\title{
Factors affecting the market adoption of cyber-security products in Energy and Electrical systems
}

\author{
Theodoros Rokkas \\ inCITES Consulting \\ Strassen Luxembourg \\ trokkas@incites.eu
}

\author{
The case of SPEAR \\ Ioannis Neokosmidis \\ inCITES Consulting \\ Strassen Luxembourg \\ i.neokosmidis@incites.eu
}

\begin{abstract}
In this paper the results of a survey that was performed aiming to identify the main factors that can affect the market adoption of a cyber-security product in the Energy and Electrical systems is presented. In more detail the survey was implemented using the Fuzzy Analytic Hierarchy Process method among experts of the H2020 R\&D project SPEAR. The survey reveals experts' vision regarding the significance of the critical factors anticipated to influence the introduction and acceptance of SPEAR or similar solutions as a technology for actors of the Energy and Electrical ecosystem.
\end{abstract}

\section{CCS CONCEPTS}

- Power and Energy • Systems Security • Computing / technology policy

\section{KEYWORDS}

Cyber-security, smart-grid, decision making, market

\section{ACM Reference format:}

Theodoros Rokkas, Ioannis Neokosmidis. 2020. Factors affecting the market adoption of cyber-security products in Energy and Electrical systems: The case of SPEAR. In Proceedings of ARES conference: 15th International Conference on Availability, Reliability and Security, August $25-28,2020$

\section{Introduction}

Production of electricity is crucial for the normal functioning of any economy and its absence can lead to a number of consequences that may have severe economic but also lethal implications. As the production of electricity is moving towards the smart-grid paradigm in which digital communications are used to monitor and

Permission to make digital or hard copies of part or all of this work for personal or classroom use is granted without fee provided that copies are not made or distributed for profit or commercial advantage and that copies bear this notice and the full citation on the first page. Copyrights for third-party components of this work must be honored. For all other uses, contact the Owner/Author

ARES 2020, August 25-28, 2020, Virtual Event, Ireland (C) 2020 Copyright is held by the owner/author(s).

ACM ISBN 978-1-4503-8833-7/20/08. https://doi.org/10.1145/3407023.3409315 react to usage changes, more parts of the power generation and delivery systems are facing new and evolving cyber-security threats.

The challenges for securing the smart grid include the development of innovative cyber security capabilities into the smart grid devices and networks allowing scalability for future scenarios. To build the secure network of tomorrow advanced tools, standards and guidelines are required. To face these needs SPEAR project [1] develops tools that provide effective detection, response and countermeasures against advanced cyber threats and attacks targeted at smart grids. Such tools are important from a user perspective, as the ability to detect different kinds of attacks concerning confidentiality, integrity and availability, as well as timely detection of these attacks are key to their business model. The SPEAR platform is a three-tier system in which each part has a different but complimentary role: the first tier builds an advanced all-in-one, open source Security Information and Event Management (SIEM) tool (SPEAR SIEM). This is designed for timeously detecting threats and attacks in smart environments. The second tier provides a rigorous forensic framework SPEAR Forensic Readiness Framework (SPEAR-FRF), aiming to assure forensic readiness in the sense that the applied network forensic strategies are deployed before a cyber-attack incident takes place. The third tier is designed in line with two major requirements of all security-oriented organisations: increasing the trust between smart grid operators and facilitating EU consensus towards confronting cyber-attacks.

Cyber security for smart grids sectors is a constantly evolving market. This trend is expected to continue in the future as more and more operators migrate their legacy networks into smart grids. That is the segment of the market that the SPEAR tool aims to enter by exploiting the results of the project.

An initial market analysis shows that there exist available tools offering the functionalities of SPEAR, however there are no specific solutions targeted to the smart grids and furthermore the available ones focus on large organizations. Most of the solutions can be customized in order to meet the needs of energy operators but at an increased cost. We believe that there is market potential in that area although the competition is heavy. It is important for new solutions that emerge in the market to identify the main 
characteristics that the new products must have in order to achieve market adoption.

In this paper we will present the work towards understanding the main factors that can affect the market adoption of SPEAR platform or others with similar characteristics. To achieve this goal, a survey using the Fuzzy Analytic Hierarchy Process (AHP) method has been conducted. The survey reveals experts' vision regarding the significance of the critical factors anticipated to influence the introduction and acceptance of SPEAR as a technology solution. The outputs can act as a guidance for stakeholders to adjust their business strategies in order to better take advantage of the commercial potentials that SPEAR is offering.

The paper is organized as follows: in section 2 a general description of the AHP decision making framework is presented, section 3 presents the application of the framework in SPEAR and section 4 presents the results. Finally, section 5 concludes the paper.

\section{Decision making using the AHP framework}

The AHP was proposed and developed by Thomas Saaty [2] in the early 1970s, mainly for military purposes, such that AHP can be considered to be a multi-criteria decision making methodology. AHP has been extensively used over the years to cover various application areas, such as education [3], engineering [4], industry [5], manufacturing [6] and resource allocation [7]. Recently, AHP has also been widely used for selecting and ranking alternatives in the field of ICT [8]-[11].

AHP is a structured technique for dealing with complex decisions, based upon a rational and comprehensive framework for decomposing an unstructured complex problem into a multi-level hierarchy of interrelated criteria, sub-criteria and decision alternatives. By incorporating judgments on qualitative and quantitative criteria, AHP manages to quantify decision-makers' preferences. The relative priorities of the criteria, sub-criteria and alternatives are finally reached by a mathematical combining of all these various judgments.

AHP is implemented performing the following steps: in the first step, the problem that will be investigated is framed (i.e., its formation articulated), while the criteria and sub-criteria contributing to the objective's satisfaction are determined through interviews and/or group discussions with experts. The multi-level hierarchy is then constructed, consisting of three levels. In the first level, the objective under investigation is shown. In this work, the factors affecting the adoption and evolution of SPEAR in general are being examined. In the next level, the criteria, $\mathrm{C}_{\mathrm{rk}}$ with $\mathrm{k}=1,2, \ldots, \mathrm{N}$ and $\mathrm{N}$ the total number of criteria, participating in the decision-making process are determined. The criteria should be general enough to incorporate several features resulting in a rough description of the objective. In the lower level, criteria are further analyzed into their sub-criteria $\mathrm{SC}_{\mathrm{rjk}}$, where $\mathrm{j}=1,2, \ldots, \mathrm{M}_{\mathrm{k}}$ and $\mathrm{M}_{\mathrm{k}}$ is the number of sub-criteria under criterion $\mathrm{k}$. Sub-criteria represent a specific feature characterizing a criterion. Identification of criteria and sub-criteria is accomplished based on the focus of their preferential independence. Once the hierarchical structure has been constructed and the criteria and sub-criteria determined, appropriate questionnaires are created and distributed to experts (step 2) for them to fill in. The procedure here is based upon systematic pairwise judgments of the experts from the second to the lowest level of the hierarchy: In each level, the criteria (subcriteria) are compared pair-wisely according to their degree of influence and based on the specified criteria in the higher level. The described comparisons are performed using the standardized nine levels scale shown in Table 1.

Table 1: Rating scale

\begin{tabular}{|c|l|l|}
\hline $\begin{array}{c}\text { Intensity of } \\
\text { importance }\end{array}$ & Definition & Explanation \\
\hline 1 & $\begin{array}{l}\text { Equal } \\
\text { importance }\end{array}$ & $\begin{array}{l}\text { The two criteria contribute } \\
\text { equally }\end{array}$ \\
\hline 3 & $\begin{array}{l}\text { Moderate } \\
\text { importance }\end{array}$ & $\begin{array}{l}\text { Experience and judgment } \\
\text { favor one of the criteria }\end{array}$ \\
\hline 5 & $\begin{array}{l}\text { Strong } \\
\text { importance }\end{array}$ & $\begin{array}{l}\text { A criterion is strongly } \\
\text { favored }\end{array}$ \\
\hline 7 & $\begin{array}{l}\text { Very strong } \\
\text { importance }\end{array}$ & $\begin{array}{l}\text { A criterion is very strong } \\
\text { dominant }\end{array}$ \\
\hline $2,4,6,8$ & $\begin{array}{l}\text { Extreme } \\
\text { importance }\end{array}$ & $\begin{array}{l}\text { A criterion is favored by at } \\
\text { least an order of } \\
\text { magnitude }\end{array}$ \\
\hline values & $\begin{array}{l}\text { Used to compromise } \\
\text { between two of the above } \\
\text { numbers }\end{array}$ \\
\hline
\end{tabular}

The set of pairwise comparisons on the $\mathrm{N}$ criteria results in an $\mathrm{N} \mathrm{x}$ $\mathrm{N}$ evaluation matrix $\mathrm{A}=\left[\mathrm{A}_{\mathrm{ij}}\right]$ in which the elements $\mathrm{A}_{\mathrm{ij}}(>0)$ represent the relative importance of criterion $\mathrm{Cr}_{\mathrm{i}}$ as compared to $\mathrm{Cr}_{j}$. It should be noted that $\mathrm{A}_{\mathrm{ii}}=1$ for all $\mathrm{i}$, while the matrix $\mathrm{A}$ is symmetrical across the main diagonal, that is $A_{j i}=1 / A_{i j}$. The same steps are followed regarding the sub-criteria of each criterion $\mathrm{k}$, and the results are summarized in a similar matrix to A, called $A_{k}$. The last step (step 3) towards the evaluation of the objectives is the estimation of the criteria and sub-criteria weights, $w_{k}$ and $s_{j k}$ respectively. This requires the calculation of the principal eigenvector $\mathbf{v}=\left[v_{k}\right] \quad\left(\right.$ or $\boldsymbol{u}_{k}=\left[u_{i k}\right]$ ) that is the eigenvector corresponding to the maximum eigenvalue $\lambda_{\max }$ (principal eigenvalue) of matrix A (or $\boldsymbol{A}_{\boldsymbol{k}}$ ). The weights of the criterion $k$ and of each of its sub-criterion $j$ are given by:

$$
\begin{aligned}
& w_{k}=\frac{v_{k}}{\sum_{i=1}^{N} v_{i}} \\
& s_{j k}=\frac{u_{j k}}{\sum_{i=1}^{M_{k}} u_{i k}}
\end{aligned}
$$

where $N$ and $M_{k}$ is the number of criteria and sub-criteria of criterion $k$ respectively.

It is well recognized that AHP can be highly subjective and inaccurate, mainly due to its inability to adequately handle the inherent uncertainty and imprecision associated with the mapping of a decision-maker's perception to exact numbers. In this case, the Fuzzy Analytic Hierarchy Process (FAHP), an extension/improvement of the AHP methodology, has been 
Factors affecting the market adoption of cyber-security products in Energy and Electrical systems: The case of SPEAR

proposed [12]-[14] as a means to address this uncertainty. Fuzzy numbers are used in order to model the relative importance of criteria and sub-criteria.

Let $\tilde{A}$ represent a fuzzified reciprocal $N \mathrm{x} N$-judgment matrix containing all pairwise comparisons between elements $i$ and $j$ for all $i, j \in(1,2, \ldots, N)$.

$$
\tilde{A}=\left[\begin{array}{cccc}
(1,1,1) & \tilde{a}_{12} & \cdots & \tilde{a}_{1 N} \\
\tilde{a}_{21} & (1,1,1) & \cdots & \tilde{a}_{2 N} \\
\vdots & \vdots & \ddots & \vdots \\
\tilde{a}_{N 1} & \tilde{a}_{N 2} & \cdots & (1,1,1)
\end{array}\right]
$$

where $\tilde{a}_{j i}=\tilde{a}_{j i}^{-1}$ and all $\tilde{a}_{i j}$ are fuzzy numbers. The use of fuzzy numbers as answers (vague comparisons), although increasing the processing complexity, provides for more accurate and meaningful results. A fuzzy weight for each criterion and sub-criterion is evaluated, while crisp weights can also be obtained through the defuzzification process.

Fuzzy numbers are a part of the fuzzy sets theory, introduced by Zadeh [15] as a modelling tool for complex systems under uncertainty. In fuzzy sets, grades of membership in $[0,1]$ are assigned to objects through a membership function $\mu_{A}(x)$. As shown in Figure 1, in the special case of triangular fuzzy numbers, the membership is defined by three real numbers, $(l, m, u)$, where $l$ is the lower limit, $m$ the most promising and $u$ the upper limit value. In the limit, $l=m=u$, fuzzy numbers become crisp numbers. Eq. (4) describes the membership function of triangular fuzzy numbers.

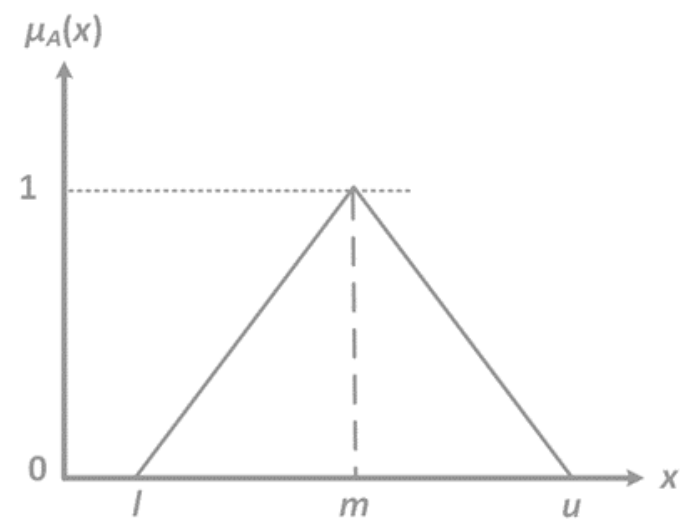

Figure 1: Triangular fuzzy numbers membership function

$$
\mu_{A}(x)=\left\{\begin{array}{c}
\frac{x-l}{m-l}, x \in[l, m] \\
\frac{u-x}{u-m}, x \in[m, u] \\
0, \text { otherwise }
\end{array}\right.
$$

Assuming that $M_{1}=\left(l_{1}, m_{1}, u_{1}\right)$ and $M_{2}=\left(l_{2}, m_{2}, u_{2}\right)$ are triangular fuzzy numbers, the operations on them can be:

$$
\text { Addition: } M_{1} \oplus M_{2}=\left(l_{1}+l_{2}, m_{1}+m_{2}, u_{1}+u_{2}\right)
$$

ARES 2020, August 25-28, 2020, Virtual Event, Ireland

$$
\begin{gathered}
\text { Multiplication: } M_{1} \otimes M_{2}=\left(l_{1} \cdot l_{2}, m_{1} \cdot m_{2}, u_{1} \cdot u_{2}\right) \\
\text { Inverse: } M_{1}^{-1}=\left(l_{1}, m_{1}, u_{1}\right)^{-1}=\left(\frac{1}{u_{1}}, \frac{1}{m_{1}}, \frac{1}{l_{1}}\right)
\end{gathered}
$$

After collecting the fuzzy judgment matrices from all decision makers, these matrices are then aggregated. An approach is to combine the fuzzy pairwise comparisons using the following algorithm [14],[17]:

$$
\begin{aligned}
l_{i j}=\min \left(l_{i j k}\right), m_{i j} & =\left(\prod_{k=1}^{K} m_{i j k}\right)^{1 / K}, u_{i j} \\
& =\max \left(u_{i j k}\right)
\end{aligned}
$$

where $\left(l_{i j k}, m_{i j k}, u_{i j k}\right)$ is the fuzzy evaluation of the sample members $k(k=1,2, \ldots, K)$. In the case of a wide range of upper and lower bandwidths (inhomogeneous evaluations), min and max operations are not appropriate, usually leading to a very large span of fuzzy numbers and allowing the aggregated fuzzy weights to exceed the predefined borders.

Therefore, the fuzzy geometric mean method [16]-[18] is used. In this case, the aggregated triangular fuzzy number of $K$ decision makers' judgment in a certain case $\left(l_{i j}, m i j, u_{i j}\right)$ is given by:

$$
\begin{gathered}
l_{i j}=\left(\prod_{k=1}^{K} l_{i j k}\right)^{1 / K}, m_{i j}=\left(\prod_{k=1}^{K} m_{i j k}\right)^{1 / K}, u_{i j} \\
=\left(\prod_{k=1}^{K} u_{i j k}\right)^{1 / K}
\end{gathered}
$$

\subsection{Consistency of pairwise comparison matrices}

In order to maintain a certain quality level of a decision, the consistency of the data should also be investigated during the analysis. It should be noted that the rank of the matrix $\boldsymbol{A}$ (or $\boldsymbol{A}_{\boldsymbol{k}}$ ) equals to 1 and $\lambda_{\max }=N$ (or $M_{k}$ ) if the pairwise comparisons are completely consistent. In this case, weights can be estimated by normalizing any of the columns or rows of $\boldsymbol{A}\left(\boldsymbol{A}_{\boldsymbol{k}}\right)$. A consistency index $(C I)$ was introduced in [2]

$$
C I=\frac{\lambda_{\max }}{N-1}
$$

where $\lambda_{\max }$ is the largest (maximum) eigenvalue and $N$ is the number of criteria. The final consistency ratio $(C R)$, showing how consistent the judgments have been relative to large samples of purely random judgments, is given by:

$$
C R=\frac{C I}{R I}
$$

where $R I$ is the random index calculated as the average $C I$ across a large number of randomly filled matrices using the scale described earlier in this section. The random indices for several values of $N$ were calculated by Saaty [19] and presented in Table 2 . 
Table 2: RI values for different values of $\mathbf{n}$

\begin{tabular}{|c|c|c|c|c|c|c|c|c|c|}
\hline $\boldsymbol{n}$ & $\mathbf{1}$ & $\mathbf{2}$ & $\mathbf{3}$ & $\mathbf{4}$ & $\mathbf{5}$ & $\mathbf{6}$ & $\mathbf{7}$ & $\mathbf{8}$ & $\mathbf{9}$ \\
\hline $\boldsymbol{R I}$ & 0 & 0 & 0.58 & 0.90 & 1.12 & 1.24 & 1.32 & 1.41 & 1.45 \\
\hline
\end{tabular}

The consistency ratio should be less than 0.1 . A $C R$ larger than the tolerable level of 0.1 demonstrates the need to exclude the pairwise comparison matrix of this respondent for further analysis so as not to affect the overall accuracy of the results.

In order to reduce the complexity, and without loss of generality, authors usually verify the consistency only for crisp matrices whose elements are the middle significant values of the triangular fuzzy numbers from the corresponding fuzzy pairwise comparison matrix [20]. This approach was used in this paper to assess the consistency of the pairwise comparison matrices.

\section{Application to SPEAR}

In order to identify the factors that will influence the adoption of SPEAR, a survey was designed with the assistance of partners in the SPEAR consortium. Following the AHP methodology, initially the set of criteria were defined after discussions with the experts among the project consortium:

- Performance: Aspects as speed and accuracy of detection, availability and scalability

- Technology / Features: Aspects as visualization, automation, integration with existing systems

- Security / Compliance: Aspects related to confidentiality and security, privacy

- Business /Strategy aspects: Market related issues

Each of these criteria was further broken down into sub-criteria, that are usually indicative attributes that can be quantified and are closely related to the criteria. As in the previous step these sets were selected after discussion with the experts.

The following paragraphs present the sets of sub-criteria for each of the criteria.

Regarding the Performance criterion, five sub-criteria were identified:

- $\quad$ Fast detection and response: Ability to detect and respond early to cyberattacks.

- Accuracy of detection: Ability to detect possible threats with high accuracy and F1 score.

- Detection of known and unknown attacks: Not only the most known cyberattacks should be detectable, but also suspicious activities that could indicate a zero-day attack.

- Availability: The overall functionality supported by the SPEAR platform should always be available.
- Scalability: The SPEAR system should be able to expand its monitoring capabilities on multiple networks and sub-networks.

For the Technology / Features criterion, five sub-criteria were identified:

- Visualization: SPEAR should visualise the security status of the network in a user-friendly manner.

- Automation: SPEAR should provide the option to automate numerous operational tasks, such as updates, snapshots and backups.

- Remote notification: SPEAR should notify operators remotely regarding ongoing security incidents.

- Integration with existing systems: SPEAR should collaborate and be compatible with existing systems in the monitored network (e.g., Syslog devices, Simple Network Management Protocol (SNMP) infrastructure).

- Information sharing: The platform will publish anonymised cybersecurity incidents in a shared repository.

For the Security / Compliance criterion, five sub-criteria were identified:

- Confidentiality / Integrity / Privacy: SPEAR data and functionalities will be protected from falsification and unauthorised access.

- Compliance with regulation: SPEAR will be compliant with the latest regulations regarding security and data protection, like GDPR.

- Security of systems: All SPEAR sub-systems should be properly secured from unauthorised access and penetration.

- Security of interfaces: The communication between sub-systems of SPEAR should be protected by encryption and proper security schemes.

- Accountability: All access attempts and operator actions should be properly recorded.

For the Business /Strategy aspects criterion, four sub-criteria were identified:

- Cost: The SPEAR solution should be offered at low cost.

- Flexible pricing: A flexible pricing scheme should be offered to clients that have small, medium or large-scale requirements scheme should be offered to clients that have small, medium or large-scale requirements.

- Creation of new business models: SPEAR will allow the Creation of new business models. 
Factors affecting the market adoption of cyber-security products in Energy and Electrical systems: The case of SPEAR

- Advantages over competition: SPEAR solution will have advantages over competing solutions.

Taking these under consideration a web-based survey was created incorporating all elements of the Fuzzy AHP framework. Experts were asked to determine the (sub)criterion of his/her preference (for every pair of (sub)criteria) and provide the upper and lower limit of their relative importance. The responses were extracted, and a tool implemented in Matlab was used to estimate the weights that signify the importance of criteria and sub-criteria.

\section{Results}

The first step towards the evaluation of the required weights is to combine/aggregate the fuzzy pairwise comparisons provided by the experts using eq. (9). As mentioned above, the consistency of the pairwise comparison matrices were examined. Six (6) of the twenty-one (21) expert's judgments were discarded as inconsistent $(\mathrm{CR}>0.1)$. Furthermore, the $\mathrm{CR}$ of the aggregate matrix is also $<0.1$, indicating "consistency". Using the aggregated fuzzy comparison matrices one can easily estimate both fuzzy and crisp weights prioritizing the criteria and sub-criteria.

\subsection{Weights of criteria}

Regarding the weights of the criteria, the results are shown in Table 3. According to the opinions of the experts, the most important criterion for SPEAR is the Performance with a weight equal to 0.43 (43\%), followed by Technology that has a weight of $0.26(26 \%)$. Security follows with a weight equal to 0.20 (20\%), while the Business criterion has the lowest weight (11\%).

Table 3: Crisp Weights of Criteria

\begin{tabular}{|l|c|}
\hline Criteria $\left(\mathbf{C}_{\mathbf{i}}\right)$ & Crisp Weight \\
\hline $\mathrm{C}_{1}:$ Performance & 0.43 \\
\hline $\mathrm{C}_{2}$ : Technology / Features & 0.26 \\
\hline $\mathrm{C}_{3}:$ Security / Compliance & 0.20 \\
\hline $\mathrm{C}_{4}:$ Business / Strategy aspects & 0.11 \\
\hline
\end{tabular}

Performance is rated higher and is clearly the most important factor that SPEAR must address according to the opinion of the experts. Users are more interested in these sets of features that will be critical for the development of the final product.

Technology and Features of the SPEAR solution receives the second higher weight as experts value the different technologies that must be adopted and implemented within the final product but lower than its performance.

Security and Compliance come in the third place, below performance and technology. An interpretation of that is that the security aspects of the developed tools are self-evident and considered to be implemented de-facto in the SPEAR tool.

The Business criterion has the lowest weight, a possible interpretation of this outcome is that because SPEAR is still in the
ARES 2020, August 25-28, 2020, Virtual Event, Ireland

development process of the different modules and there is not yet a product with high TRL and thus close to commercialization, experts are more concerned about the criteria associated with the development of the final product. At that stage of the project, the business aspects of SPEAR are not yet mature and considered as important as the other criteria.

A different way of interpretation of the results is that the decision making does not always imply a discrete choice between alternatives, but could also refer to probabilities, possibilities or considerations concerning opportunities vs. risks. The usage of fuzzy numbers could then be taken to guarantee the minimum and maximum values. An $\alpha$-cut can also be taken into account in order to define narrower lower and upper limits of the relevant weightings based on risk considerations.

Taking into consideration the fuzzy weights (illustrated in Figure 2) we observe that Performance is the clear choice of experts among the criteria and although it presents the higher uncertainty, it also presents limited overlap with the Technology criterion. Technology and Security follow in the preferences of experts having a partial overview and similar uncertainties, as a result, the order of these two is not so clear and there is a change of mutual change in ranking. However, in order to calculate the probability of rank reversal one should resort to either Monte Carlo simulations or closed-form approximations, which are somewhat out of the scope of this report. The Business criterion is ranked as the least important with the lowest uncertainty among the others while the overlap with Security is marginal.

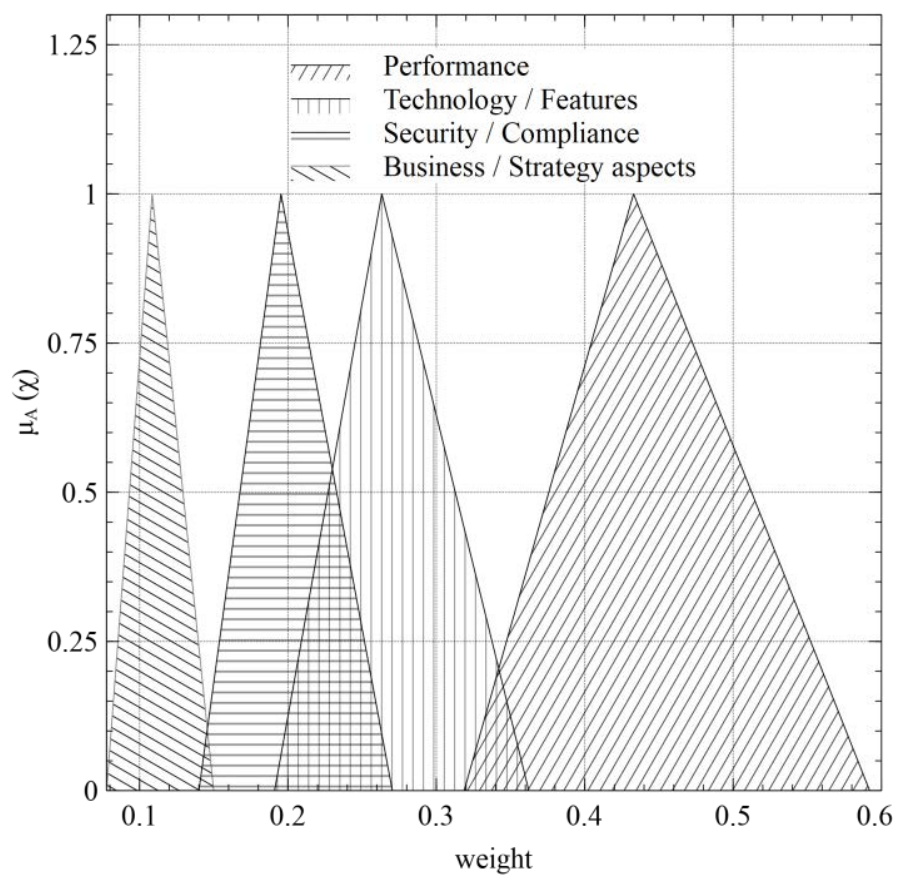

Figure 2: Fuzzy evaluation of Criteria 


\subsection{Weights of Performance sub-criterion}

It is also interesting to examine the weights of the sub-criteria under each criterion. For the Performance sub-criterion, the results are presented in Table 4. We see that four out of five sub-criteria with the exclusion of scalability have similar weights. According to experts, preferences Availability and Accuracy of Detection have equal weights of $(25 \%)$, followed by Fast Detection $(23 \%)$ and Detection (22\%) of all threats. All these sub-criteria are of equal importance and desired as characteristics that must be implemented in the developed solution. Scalability comes last with only (6\%) for the Performance criterion.

Table 4: Crisp Weights of Performance sub-criterion

\begin{tabular}{|l|c|}
\hline Sub-Criteria $\left(\mathbf{S C}_{\mathbf{i j}}\right)$ & Crisp Weight \\
\hline $\mathrm{SC}_{11}$ : Fast detection and response & 0.23 \\
\hline $\mathrm{SC}_{12}:$ Accuracy of detection & 0.25 \\
\hline $\begin{array}{l}\mathrm{SC}_{13}: \text { Detection of known and } \\
\text { unknown attacks }\end{array}$ & 0.22 \\
\hline $\mathrm{SC}_{14}:$ Availability & 0.25 \\
\hline $\mathrm{SC}_{15}:$ Scalability & 0.06 \\
\hline
\end{tabular}

Examining the fuzzy weights as these are illustrated in Figure 3, we see that the four sub-criteria overlap and since they have similar uncertainties, it is difficult to classify them. Scalability ranks last with no overlap and with the lowest uncertainty among the other sub-criteria. The understanding of these results is that experts value almost equally the four sub-criteria and it is not clear what is the most important among them. As already stated, an interpretation of this outcome is that the developed solution must meet all these Performance characteristics in order to be adopted by the users.

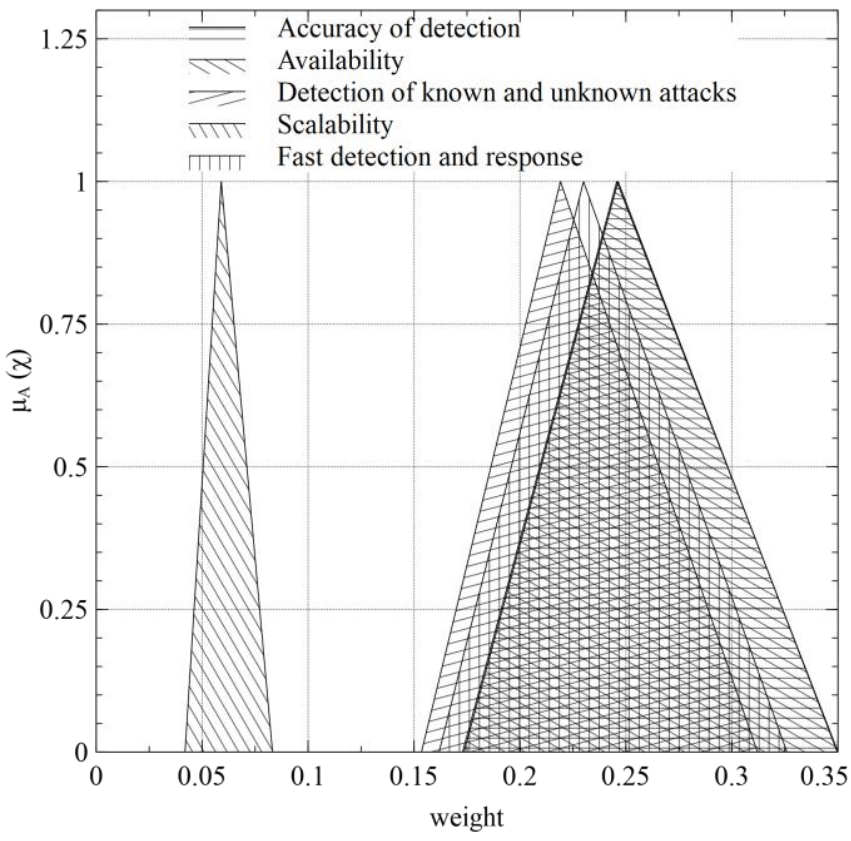

Figure 3: Fuzzy evaluation of Performance Sub-criterion

\subsection{Weights of Technology sub-criterion}

Continuing to the Technology criterion, the results for the subcriteria are presented in Table 6. Integration with existing systems has the highest weight (33\%), followed by Automation (21\%). Visualization (18\%) and Remote Notification (16\%) rank third and fourth with similar weights, while Information Sharing ranks in the last place with $11 \%$.

Integration with existing systems is the feature that experts valuate as the most desirable that SPEAR must have. This is an important feature since operators have existing systems in operation and do not wish to make any changes to them. Automation, visualization and remote notification have similar significance for experts.

Table 5: Crisp Weights of Technology sub-criterion

\begin{tabular}{|l|c|}
\hline Sub-Criteria $\left(\mathbf{S C}_{\mathbf{i j}}\right)$ & Crisp Weight \\
\hline $\mathrm{SC}_{21}$ : Visualization & 0.18 \\
\hline $\mathrm{SC}_{22}$ : Automation & 0.21 \\
\hline $\mathrm{SC}_{23}$ : Remote notification & 0.16 \\
\hline $\mathrm{SC}_{24}$ : Integration with existing systems & 0.33 \\
\hline $\mathrm{SC}_{25}$ : Information sharing & 0.11 \\
\hline
\end{tabular}

Examining the fuzzy weights, we see that Integration with existing systems receives both the highest weight and highest uncertainty. However, it has only partial overlap with automation. The next three sub-criteria have similar uncertainties and high overlap, suggesting that characteristics of Remote notification, Automation and Visualization are desirable features but with no strong preference among the experts. Information sharing although it appears to have the lowest mean value it overlaps with Remote Notification.

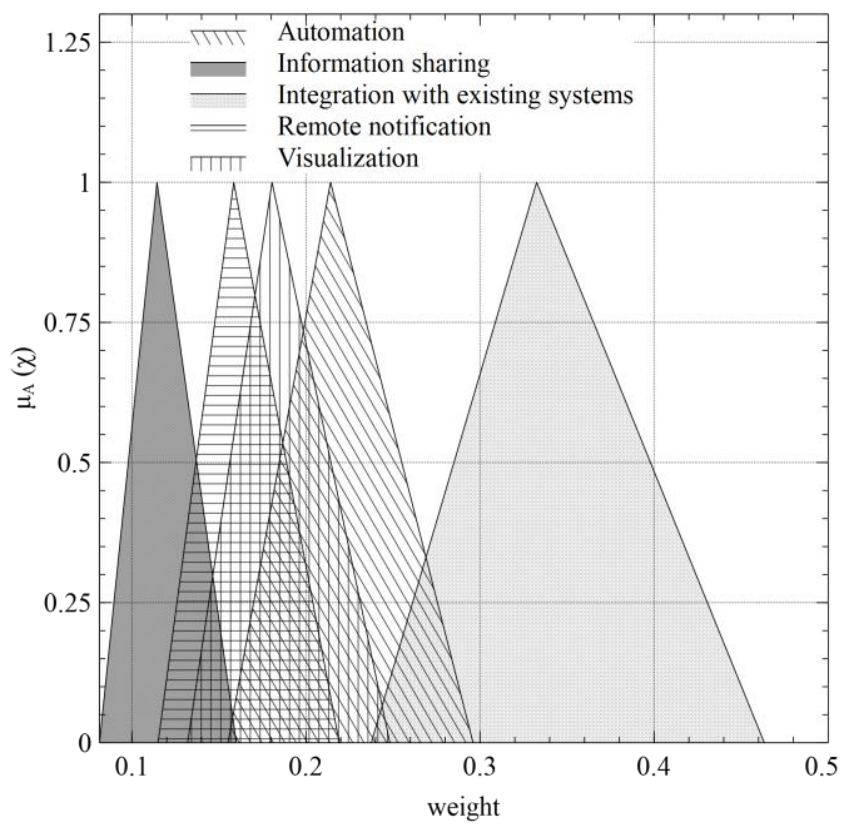

Figure 4: Fuzzy evaluation of Technology Sub-criterion 
Factors affecting the market adoption of cyber-security products in Energy and Electrical systems: The case of SPEAR

\subsection{Weights of Security sub-criterion}

Regarding the Security criteria, the results for both the crisp and fuzzy sub-criteria weights are presented in Table 6. Security of systems has the highest weight $(40 \%)$ among the sub-criteria highlighting the need for securing all the developed systems. Security of interfaces and Confidentiality / Integrity follow with weights of $21 \%$ and $19 \%$ respectively. These are features that are also considered essential for the SPEAR. Compliance with regulation and accountability have the lowest of weights with $13 \%$ and $8 \%$ respectively.

Table 6: Crisp Weights of Security sub-criterion

\begin{tabular}{|l|c|}
\hline Sub-Criteria $\left(\mathbf{S C}_{\mathrm{ij}}\right)$ & Crisp Weight \\
\hline SC31: Confidentiality / Integrity / & \\
Privacy & 0.19 \\
\hline SC32: Compliance with regulation & 0.13 \\
\hline SC33: Security of systems & 0.40 \\
\hline SC34: Security of interfaces & 0.21 \\
\hline SC35: Accountability & 0.08 \\
\hline
\end{tabular}

Considering the fuzzy weights that are illustrated in Figure 5, one can see that the Security of Systems ranks first and even though it presents high uncertainty, it has very limited overlap with the Security of Interfaces sub-criterion. The landscape for the subcriterion with the second highest weighting is not so clear: Security of Interfaces and Confidentiality follow having similar weights and uncertainties, so it is not clear which is most important for experts. Compliance with regulation has slightly lower mean weight and similar uncertainty with the previous sub-criteria and partial overlaps with both of them. Accountability receives the lowest weight and also the lowest uncertainty among this group of subcriteria.

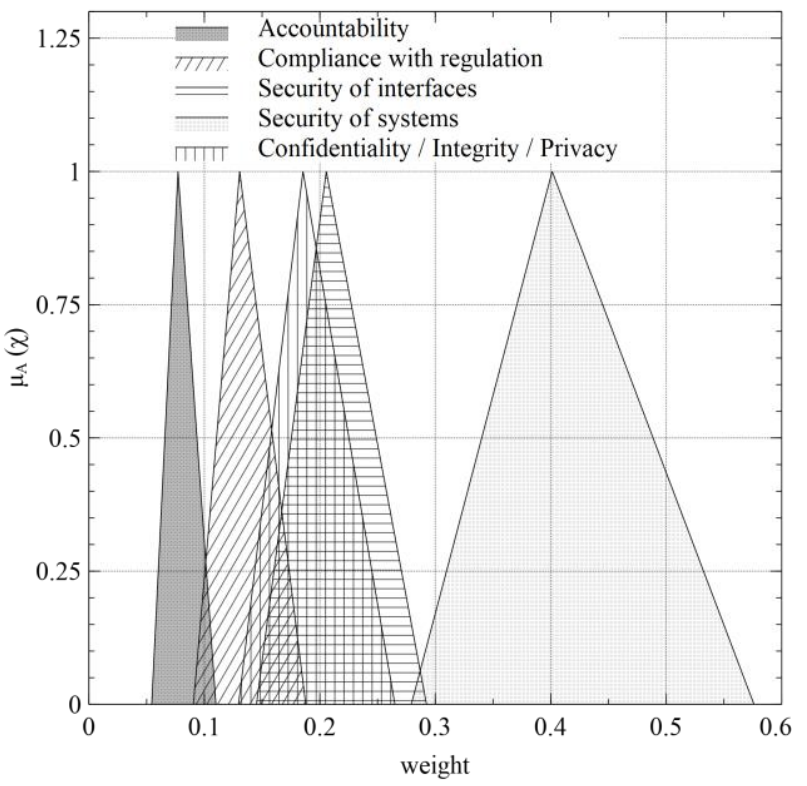

Figure 5: Fuzzy evaluation of Security Sub-criterion
ARES 2020, August 25-28, 2020, Virtual Event, Ireland

\subsection{Weights of Business sub-criterion}

The results for the crisp weights are illustrated in Table 7. Advantages over the competition is perceived as the sub-criterion with the highest importance and receives a weight of (48\%).

Since the market is dominated by a number of competitive products, SPEAR must offer advantages over the other commercially available solutions in order to find its place on the market. Flexible pricing receives the second highest weight (27\%), most of the commercially available products are targeted to big companies and the pricing is tailored to their needs.

Table 7: Crisp Weights of Business sub-criterion

\begin{tabular}{|l|c|}
\hline Sub-Criteria $\left(\mathbf{S C}_{\mathbf{i j}}\right)$ & Crisp Weight \\
\hline $\mathrm{SC}_{41}$ : Cost & 0.15 \\
\hline $\mathrm{SC}_{42}$ : Flexible pricing & 0.27 \\
\hline $\mathrm{SC}_{43}$ : Creation of new business models & 0.11 \\
\hline $\mathrm{SC}_{44}:$ Advantages over competition & 0.48 \\
\hline
\end{tabular}

The requirement for flexible pricing that adjusts to their need is essential, especially for small operators and power generators since there are not available products targeted specifically to them. Cost follows with a weight of $15 \%$, it seems that experts are willing to pay for SPEAR if the product fits their needs without thinking high cost as a barrier. Of course, we have to consider that the cost should be at the level of other competitive solutions that are offered. Finally, experts do not valuate that SPEAR will create new business models in the area.

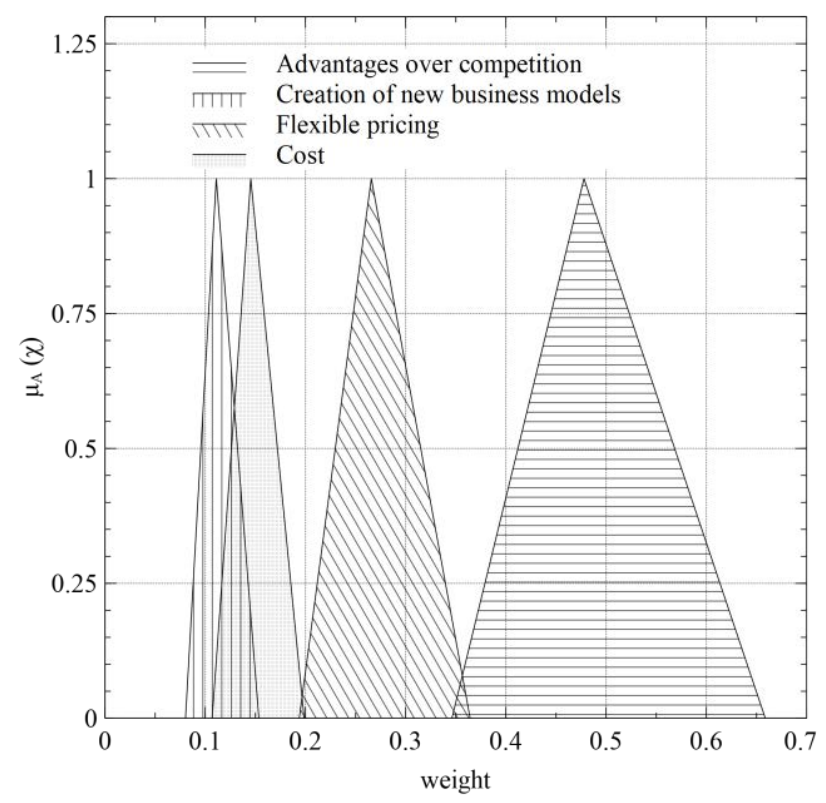

Figure 6: Fuzzy evaluation of Business Sub-criterion

Considering the fuzzy weights (Figure 6), we see more or less that the situation for this Sub-criteria is very similar to the crisp weights. 
With the exception of Cost and Creation of new business models for which there is a partial overlap that makes difficult to rank these two sub-criteria, the situation for the most significant factors is clear and with limited overlap. Advantage over competition has the highest weight and although it has the highest uncertainty, it has a clear advantage over the Flexible Pricing.

\section{Conclusions}

After the processing of expert's responses and the market analysis of the relevant market, the following conclusions can be drawn:

The most important criterion that is essential for the adoption of SPEAR is the achieved performance of the developed tool. Several factors must be considered that have similar significance such as fast detection and response, accuracy of detection, availability, and detection of all type of threats. Scalability is not considered of equal importance but must be also considered.

Regarding the implemented technology features, integration with existing systems seems to hold the most important place among the different factors. Automation and Visualization must be implemented along with Remote notification and Information Sharing.

Security between the different systems is the most important factor followed by the security of Interfaces. Experts also recognize that the developed tool must provide confidentiality and privacy, and compliance with the regulatory framework. From the relevant factors, experts prioritize the least the accountability.

The business aspects of the developed platform are not yet considered as important factors at this stage of the project. Among the identified factors, the advantages over the competitive solutions have the priority along with the ability of flexible pricing.

We hope that the analysis presented in this paper will be a valuable tool for all the people around the security of the Smart Grid ecosystem: researchers, academia, energy operators, small producers, and other involved stakeholders. It provides a list with all the significant factors that can affect the adoption of SPEAR (or similar solutions). By identifying these factors, stakeholders can create their development strategy.

\section{ACKNOWLEDGMENTS}

The research leading to these results has received funding from the European Union's Horizon 2020 research and innovation programme under grant agreement 787011.

\section{REFERENCES}

[1] https://www.spear2020.eu/

[2] T. L. Saaty, "A scaling method for priorities in hierarchical structures," Journal of Mathematical Psychology, vol. 15, pp. 234-281, 1977.

[3] A. M. A. Bahurmoz, "The analytic hierarchy process at DarAl-Hekma, Saudi Arabia," Interfaces, vol. 33, pp. 70-78, 2003.

[4] A. Kengpol and C. O'Brien, "The development of a decision support tool for the selection of advanced technology to achieve rapid product development," International Journal of Production Economics, vol. 69, pp. 177-191, 2001.

[5] G. Noci and G. Toletti, "Selecting quality-based programmes in small firms: A comparison between the fuzzy linguistic approach and the analytic hierarchy process," International Journal of Production Economics, vol. 67, pp. 113-133, 2000.

[6] M. M. Albayrakoglu, "Justification of New Manufacturing Technology: A Strategic Approach Using the Analytical Hierarchy Process," Production and Inventory Management Journal, First Quarter, vol. 37, pp. 71-76, 1996.

[7] T. L. SAATY, The analytic hierarchy process: planning, priority setting, resource allocation. New York: McGraw-Hill International Book Co., 1980.

[8] G. Dede, et al., "Towards a Roadmap for Future Home Networking Systems: An Analytical Hierarchy Process Approach," IEEE Systems Journal, vol. 5, pp. 374384, 2011.

[9] G. Dede, et al., "Evaluation of Optical Wireless Technologies in Home Networking: An Analytical Hierarchy Process Approach," IEEE/OSA Journal of Optical Communications and Networking, vol. 3, pp. 850-859, 2011.

[10] S. Nikou, et al., "Analytic Hierarchy Process (AHP) Approach for Selecting Mobile Service Category (Consumers' Preferences)," in 2011 10th International Conference on Mobile Business, 2011, pp. 119-128.

[11] S. Qingyang and A. Jamalipour, "Network selection in an integrated wireless LAN and UMTS environment using mathematical modeling and computing techniques," IEEE Wireless Communications, vol. 12, pp. 42-48, 2005.

[12] D.-Y. Chang, "Applications of the extent analysis method on fuzzy AHP," European Journal of Operational Research, vol. 95, pp. 649-655, 1996.

[13] P. J. M. van Laarhoven and W. Pedrycz, "A fuzzy extension of Saaty's priority theory," Fuzzy Sets and Systems, vol. 11, pp. 229-241, 1983/01/01 1983.

[14] T.-H. Chang and T.-C. Wang, "Using the fuzzy multi-criteria decision making approach for measuring the possibility of successful knowledge management," Information Sciences, vol. 179, pp. 355-370, 2009.

[15] L. A. Zadeh, "Fuzzy sets," Information and Control, vol. 8, pp. 338-353, 1965.

[16] J. J. Buckley, "Ranking alternatives using fuzzy numbers," Fuzzy Sets and Systems, vol. 15, pp. 21-31, 1985/02/01 1985.

[17] J. J. Buckley, "Fuzzy hierarchical analysis," Fuzzy Sets and Systems, vol. 17, pp. 233-247, 1985/12/01 1985

[18] J. J. Buckley, et al., "Fuzzy hierarchical analysis revisited," European Journal of Operational Research, vol. 129, pp. 48-64, 2001.

[19] T. L. Saaty and M. S. Ozdemir, "Why the magic number seven plus or minus two," Mathematical and Computer Modelling, vol. 38, pp. 233-244, 2003.

[20] N.-F. Pan, "Fuzzy AHP approach for selecting the suitable bridge construction method," Automation in Construction, vol. 17, pp. 958-965, 2008. 